\title{
Brown adipose tissue-specific insulin receptor knockout shows diabetic phenotype without insulin resistance
}

\author{
Carmen Guerra, ${ }^{1}$ Paloma Navarro, ${ }^{2}$ Angela M. Valverde, ${ }^{2}$ Monica Arribas, ${ }^{2}$ \\ Jens Brüning, ${ }^{3}$ Leslie P. Kozak, ${ }^{1}$ C. Ronald Kahn, ${ }^{3}$ and Manuel Benito ${ }^{2}$ \\ 1Jackson Laboratory, Bar Harbor, Maine, USA \\ ${ }^{2}$ Departamento de Bioquimica y Biologia Molecular, Facultad de Farmacia, Universidad Complutense, Madrid, Spain \\ ${ }^{3}$ Joslin Diabetes Center, Harvard Medical School, Boston, Massachusetts, USA \\ Address correspondence to: Manuel Benito, Departamento de Bioquímica y Biologia Molecular, \\ Facultad de Farmacia, Universidad Complutense, Madrid 28040, Spain. \\ Phone: 34-91-3941777; Fax: 34-91-3941779; E-mail: benito@eucmax.sim.ucm.es.
}

Carmen Guerra and Paloma Navarro contributed equally to this work.

Received for publication April 23, 2001, and accepted in revised form September 5, 2001.

\begin{abstract}
Although insulin regulates metabolism in both brown and white adipocytes, the role of these tissues in energy storage and utilization is quite different. Recombination technology using the Cre-loxP approach allows inactivation of the insulin receptor in a tissue-specific manner. Mice lacking insulin receptors in brown adipocytes show an age-dependent loss of interscapular brown fat but increased expression of uncoupling protein-1 and -2. In parallel, these mice develop an insulin-secretion defect resulting in a progressive glucose intolerance, without insulin resistance. This model provides direct evidence for not only a role for the insulin receptors in brown fat adipogenesis, the data also suggest a novel role of brown adipose tissue in the regulation of insulin secretion and glucose homeostasis.

J. Clin. Invest. 108:1205-1213 (2001). DOI:10.1172/JCI200113103.
\end{abstract}

\section{Introduction}

Type 2 diabetes (non-insulin-dependent diabetes mellitus or NIDDM) is a polygenic disease affecting over $5 \%$ of the population in Western societies and is characterized by defects in both insulin secretion and insulin action $(1,2)$. The first detectable defect in type 2 diabetes is peripheral insulin resistance in muscle, fat, and liver $(3,4)$. Several acquired molecular alterations have been shown to contribute to insulin resistance, including a widespread decrease in the insulin receptor number, a decrease in receptor tyrosine kinase activity, low expression of insulin receptor substrate-1 (IRS-1), decreases of IRS-1 phosphorylation, and defects in glucose transporter translocation (5-8).

The ability to modify genes in a mouse has allowed us and others to create, in a general and a tissue-specific fashion, animal models in which we can assess the role of insulin receptor-mediated (IR-mediated) signaling. Mice with a global knockout of the IR die within 4-5 days after birth due to a severe ketosis $(9,10)$, while IRS-1 knockout mice exhibit a moderate growth retardation and mild glucose intolerance $(11,12)$ and IRS-2 knockout mice are of normal weight but exhibit severe glucose intolerance and impaired insulin secretion due to a beta-islet hypoplasia $(13,14)$. Doubly heterozygous IR/IRS- $1^{+/}$show insulin resistance, and about $50 \%$ develop diabetes at 6 months (15).This phenotype is further exaggerated in the triple heterozygous IR/IRS-1/IRS-2 ${ }^{+/-}$knockout mouse (16).

Inactivation of a gene by the standard homologous recombination technology produces deletion of a func- tional gene in all cells of the body. Use of the Cre-loxP system to produce a conditional mouse allows development of mice with tissue- or cell-specific gene inactivation, as well as with other types of regulated gene inactivation. The Cre-loxP system involves an enzyme of $38 \mathrm{kDa}$ called Cre recombinase from bacteriophage P1. This enzyme removes the DNA between 34-bp short recognition sequences called loxP sites. The availability of transgenic mice with cell- or tissue-specific promoters is the only limitation for this achievement. Several tissue-specific expressions of Cre in transgenic mice have already been used to inactivate the IR in a tissue-specific manner. The muscle-specific IR knockout (MIRKO) mouse exhibits several of the metabolic alterations seen in NIDDM, such as elevated triglycerides and free fatty acids, without displaying glucose intolerance (17), while mice with a tissue-specific knockout of the IR in the liver exhibit hyperglycemia at a young age and alterations in liver growth and function (18). Mice with a tissue-specific knockout of the IR in pancreatic $\beta$ cells exhibit an insulin secretory defect similar to that observed in type 2 diabetes, resulting in a progressive impairment of glucose tolerance (19), while mice with a tissue-specific knockout in the brain exhibit hyperphagia, mild obesity, and reduced fertility (20). In the present study, we have assessed the role of functional IRs in brown adipose tissue (BAT) using the Cre under the uncoupling protein1 (UCP-1) promoter. Mice lacking IRs in this tissue show a decrease in interscapular brown fat mass and a progressive decrease in glucose tolerance due to an 
insulin-secretion defect. This model not only provides direct evidence for the role of the IR in the adipogenesis of brown fat, but also points out a previously unrecognized role of BAT in control of insulin secretion and glucose homeostasis.

\section{Methods}

UCP-1-Cre transgenic mice generation. The UCP-1-Cre transgene vector was constructed by inserting the 2.4$\mathrm{kb}$ EcoRI fragment of the pTZCreN, which includes the Cre cDNA and the nuclear localization signals, close to the PstI (BglI) site of the first exon of the UCP-1 gene; $8.4 \mathrm{~kb}$ of the $5^{\prime}$ UTR from the UCP-1 gene was included in the construct to assure specificity, noradrenergic regulation, and a high level of expression. We also included a 4.3-kb HindIII fragment of the UCP-1 gene that includes part of the exon 3 and the exons 4,5 , and 6 downstream of the Cre cDNA. The UCP-1-Cre transgene was verified by sequencing.

The 15-kb linearized UCP-1-Cre DNA construct was isolated from the $\mathrm{pBS} \mathrm{SK}^{+}$vector by digestion with NotI/XhoI and purified by electrophoresis. The transgenic UCP-1-Cre mouse was generated by pronuclear microinjection of the linear DNA into $\mathrm{FVB} / \mathrm{NJ}$ zygotes at The Jackson Laboratory (Bar Harbor, Maine, USA).

The genotypes of the offspring were analyzed from tail DNA by Southern blot analysis or PCR. For Southern blot analysis, tail DNA was digested with EcoRI and processed as described (21). The Cre cDNA and the 780-bp PstI fragment for UCP-1 were used as ${ }^{32} \mathrm{P}$ probes in the hybridization. The hybridization was carried out as previously indicated (22). For PCR, $100-200 \mathrm{ng}$ of tail DNA were amplified through 30 cycles $\left(40\right.$ seconds, $94^{\circ} \mathrm{C}$; 40 seconds, $60^{\circ} \mathrm{C}$; and 1 minute, $75^{\circ} \mathrm{C}$ ) on a thermal cycler. Three primers were used for the UCP-1-Cre mice. A forward $5^{\prime}$ UCP-1 primer on exon 1 (7,496 bp) (5'-GTCTGCACTGGCACTACCTA- $\left.3^{\prime}\right)$ and two reverse $3^{\prime}$ primers, one specific for Cre ( $5^{\prime}$-CCATCGCTCGACCAGTTTAGT- $\left.3^{\prime}\right)$ and the other for the intron 1 on the UCP-1 gene (7,782 bp) ( $5^{\prime}$-GGAGAAGCCTCATCA ATGTCA-3').

Genotyping of the IRloxP transgenic mice. Genotyping of the mice was done by PCR. Tail DNA (100-200 ng) was amplified 30 cycles $\left(40\right.$ seconds, $94^{\circ} \mathrm{C} ; 40$ seconds, $60^{\circ} \mathrm{C}$; and 1 minute, $75^{\circ} \mathrm{C}$ ) on a thermal cycler. Two primers flanking the loxP site behind exon 4 of the IR were used: the forward primer ( $5^{\prime}$-GATGTACACCCCATGTCTG-3 ${ }^{\prime}$ ) and the reverse primer $\left(5^{\prime}\right.$-CTGAATAGCTGAGACCACAG-3'). A 320-bp band was obtained for the floxed allele or a 280-bp band for the wild-type allele. $R T$-PCR analysis of IR gene expression. Total RNA $(2 \mu \mathrm{g})$ isolated from BAT or isolated islets was used as template for the RT-PCR assay using the following primers. The reverse transcriptase was carried out using a reverse primer specific for IR on exon 6: 5'-GTGATGGTGAGGTTGTGTTTGCTC- $3^{\prime}$. The reaction was performed at $48^{\circ} \mathrm{C}$ for 30 minutes using the TaqMan Gold RT-PCR Kit from Applied Biosystems (Branchburg, New Jersey, USA). The PCR was performed with $5 \mu \mathrm{l}$ from the reac- tion mixture with the following set of primers: a forward primer on exon 3, 5'-GCTGCACAGCTGAAGGCCTGT-3', and a reverse primer on exon 5, 5'-CTCCTCGAATCAGATGTAGCT- $3^{\prime}$. The PCR cycling used was $58^{\circ} \mathrm{C}$ for annealing, a $72^{\circ} \mathrm{C}$ extension, and 34 cycles.

Northern blot analysis. Mice were sacrificed by cervical dislocation, and the tissues were frozen immediately in liquid nitrogen. Total RNA from different tissues or isolated islets obtained by the guanidinium-thiocyanate method (21) was analyzed as previously described (22). The membranes were hybridized overnight at $42^{\circ} \mathrm{C}$ with different cDNA ${ }^{32}$ P-probes - UCP-1, UCP-2, fatty acid synthase (FAS), glycerol 3-phosphate dehydrogenase (GP3D), malic enzyme (ME), glucose transporter Glut- 4 , CEBP- $\alpha$, PPAR $\gamma$, insulin, $18 \mathrm{~S}$ rRNA - and exposed to film in intensifying plate screens. Densitometric analysis of the autoradiograms were performed using a GS-710 Imaging Densitometer (Bio-Rad Laboratories, Hercules, California, USA).

Immunoprecipitations, Western blot analysis, and phosphatidylinositol 3-kinase activity. Five units of regular human insulin (Novo Nordisk A/S, Bagsvaerd, Denmark) were injected as a bolus into the inferior vena cava of anesthetized mice. The liver was removed 1 minute after insulin injection. WAT, BAT, and the quadriceps muscle were removed at $2.5,3$, and $3.5 \mathrm{~min}$ after injection, respectively. Tissues were homogenized as described (17). Equal amounts of protein (3-5 mg) were immunoprecipitated at $4^{\circ} \mathrm{C}$ with the anti-IR $\beta$-chain or anti-phospho tyrosine (anti-Tyr (P)) mAb, and the immune complexes were collected on antimouse Ig agarose beads. Immunoprecipitates were analyzed by SDS-PAGE followed by Western blot analysis (23). Phosphatidylinositol (PI) 3-kinase activity was measured in the anti-Tyr $(\mathrm{P})$ immunoprecipitates by in vitro phosphorylation of phosphatidylinositol (23).

Analytical procedures. Serum obtained from fasted or fed animals was analyzed for insulin by radioimmunoassay using rat insulin as the standard (Linco Research Co., St. Charles, Missouri, USA), triglyceride using a colorimetric enzyme assay kit (BoehringerMannheim GmbH, Mannheim, Germany), and free fatty acid using the free fatty acids, half-micro test (Boehringer-Mannheim $\mathrm{GmbH}$ ). Blood glucose level was determined in fed and fasted control and BAT-specific IR knockout (BATIRKO) mice using an automatic monitor (Boehringer-Mannheim $\mathrm{GmbH}$ ).

Glucose tolerance tests were performed on animals that had been fasted overnight, using an intraperitoneal glucose injection of $2 \mathrm{~g} / \mathrm{kg}$ body weight. Blood glucose values were determined from whole blood obtained from the tail at $0,30,60$, and 120 minutes, using an automatic monitor (Boehringer-Mannheim $\mathrm{GmbH}$ ). Insulin-tolerance tests were performed on fed animals injected with $1 \mathrm{U} / \mathrm{kg}$ body weight of human insulin (Novo Nordisk A/B) into the peritoneal cavity. Blood glucose values were measured at $0,15,30$, and 60 minutes, using an automatic monitor. Results were expressed as percentage of initial blood glucose concentration. 
For acute insulin release experiments, mice were fasted overnight, and then injected intraperitoneally with glucose ( $3 \mathrm{~g} / \mathrm{kg}$ body weight). Whole blood was collected from the tail vein at 0,5 , and 10 minutes and immediately centrifuged. Insulin content in plasma was determined by ELISA with a mouse insulin standard (Crystal Chem, Chicago, Illinois, USA).

Islet isolation, insulin secretion, and islet insulin content. For insulin-secretion experiments in vitro, islets were isolated using the intraductal collagenase technique. Islets were handpicked under a stereomicroscope (Wild M3B, Heerbrugg, Switzerland) and used for RNA extraction or insulin-secretion experiments. For secretion experiments, islets of similar sizes (100-150 mm) were handpicked from control and BATIRKO mice. All experiments were carried out with islets from a single harvest pool. Islets were incubated in $1 \mathrm{ml}$ of a basal buffer $(115$ $\mathrm{mM} \mathrm{NaCl}, 24 \mathrm{mM} \mathrm{NaHCO} 3,5 \mathrm{mM} \mathrm{KCl}, 1 \mathrm{mM}$ $\mathrm{MgCl}_{2} \cdot 6 \mathrm{H}_{2} \mathrm{O}, 1 \mathrm{mM} \mathrm{CaCl} 2 \cdot 2 \mathrm{H}_{2} \mathrm{O}$, with $5 \mathrm{mg} / \mathrm{ml} \mathrm{BSA}$ ) supplemented with glucose $16.7 \mathrm{mM}$. After the incubation, the medium was collected and stored at $-20^{\circ} \mathrm{C}$ for RIA of insulin. The islets were extracted in acid ethanol and stored at $-20^{\circ} \mathrm{C}$ for measurement of insulin content by RIA.

Immunohistochemical analysis. Pancreata were rapidly dissected, fixed in Bouin's solution for several hours, and then placed in PBS, pH 7.4. Paraffin-embedded pancreata were sectioned and stained for $\beta$ cells using guinea pig anti-insulin Ab (1:100, DAKO Corp., Carpinteria, California, USA) and incubated overnight at $4^{\circ} \mathrm{C}$. Secondary $\mathrm{Ab}$ (1:500, rabbit antiguinea pig IgG heavy and light chains; Zymed Laboratories Inc., San Francisco, California, USA) was incubated for 30 minutes at room temperature. The detection of $\beta$ cells was done using a $\mathrm{ABC}$ peroxidase staining and 3,3-diaminobenzidine (DAB) substrate kit (Pierce Chemical Co., Rockford, Illinois, USA).

Statistical analysis. All values are expressed as mean plus or minus SEM. Statistical analyses were carried out using a two-tailed Student's unpaired $t$ test, and the null hypothesis was rejected at the 0.05 level.

\section{Results}

Creation of the UCP 1-Cre transgenic mice. To produce a mouse in which IR was selectively inactivated in BAT, we have used the UCP-1 gene promoter, a gene that is expressed exclusively in BAT, to direct the expression of the Cre cDNA. The UCP-1-Cre transgene vector was constructed as described in Methods, and its sequence was verified by complete sequencing (Figure 1a). Two transgenic lines (lines 13 and 14) of UCP-1-Cre mice with a similar copy number of the transgene were identified by Southern blot and PCR analysis. Analysis of the mRNA for the Cre recombinase by Northern blotting revealed that both lines had a restricted expression of Cre in BAT as compared with brain, white adipose tissue, liver, or skeletal muscle (Figure 1b), although the levels of the Cre mRNA were much lower in line 13 than in line 14. For efficient production of the knockout mice, we therefore used line 14 of the UCP-1-Cre mice and always as hemizygous $(\mathrm{T} /+)$.

Creation of the BAT-specific IR knockout mice. To create specific BAT inactivation of the IR, we bred IRLox/+ (17-20) and UCP-1-Cre (T/+) obtaining the $\mathrm{F}_{1}$ out of four individual crosses at the expected ratio [nine $\mathrm{IR}^{\mathrm{Lox} /+} ; \mathrm{UCP}-1-\mathrm{Cre}(\mathrm{T} /+)$, ten $\mathrm{IR}^{\mathrm{Lox} /+}$, eight UCP-1-Cre $(\mathrm{T} /+)$, and nine wild-type]. Interbreeding of the $\mathrm{F}_{1}$ generation $\mathrm{IR}^{\mathrm{Lox} /+} ; \mathrm{UCP}-1-\mathrm{Cre}(\mathrm{T} /+)$ and $\mathrm{IR}^{\mathrm{Lox} /+}$ was performed to create a BATIRKO mouse. These mice $\left[\mathrm{F}_{2}\right.$ generation IR ${ }^{\text {Lox/Lox; }}$ UCP-1-Cre $(\mathrm{T} /+)$ ] were obtained at

\section{Figure 1}

Structure and expression of the UCP1-Cre transgene. (a) The Cre cDNA sequence was included at the Bglll site of the first exon of UCP-1 gene. The length of $5^{\prime}$ UTR of the UCP- 1 gene was $8.4 \mathrm{~kb}$. Exons 3, 4, 5, and 6 of the UCP-1 gene were also incorporated downstream of the Cre cDNA sequence. (b) Representative Northern blot analysis of total RNA isolated from several tissues of the two transgenic lines (lines 13 and 14) of mice carrying a UCP-1-Cre DNA construct described in a and C57BL/6J (B6) control mouse. Blots were hybridized with a full-length Cre cDNA probe. a

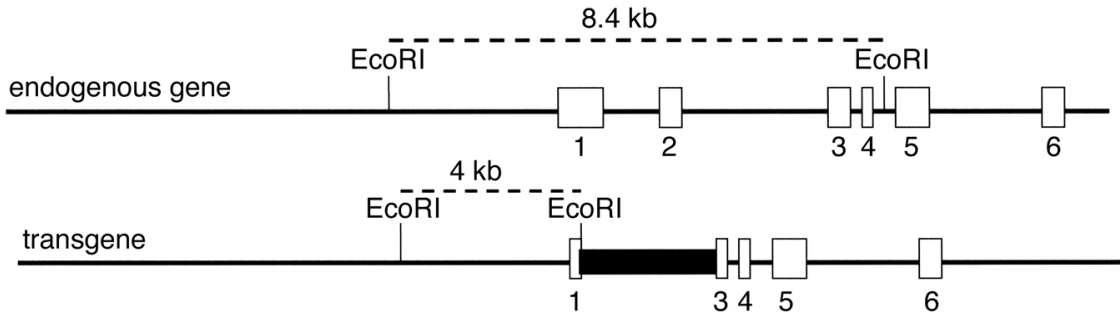

b Line 13

Line 14

Control

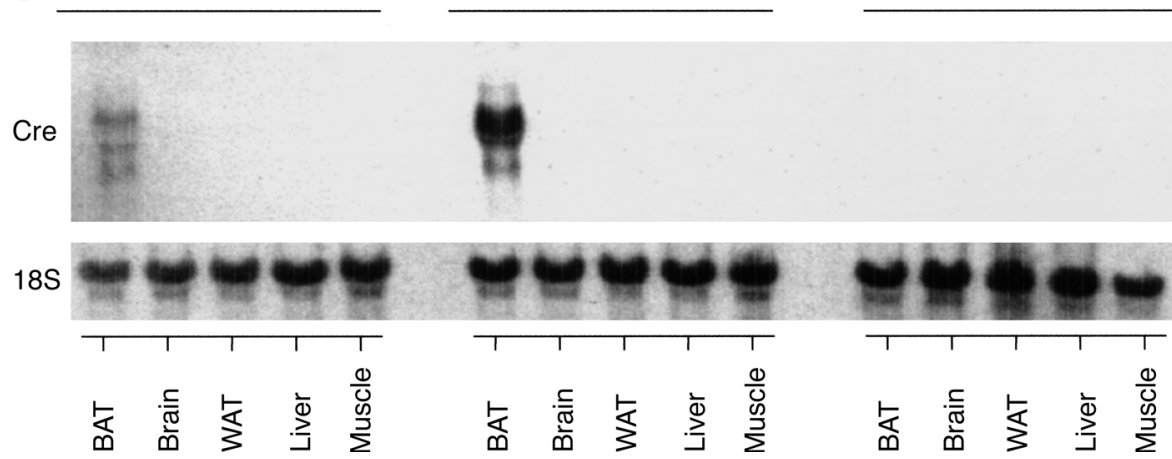




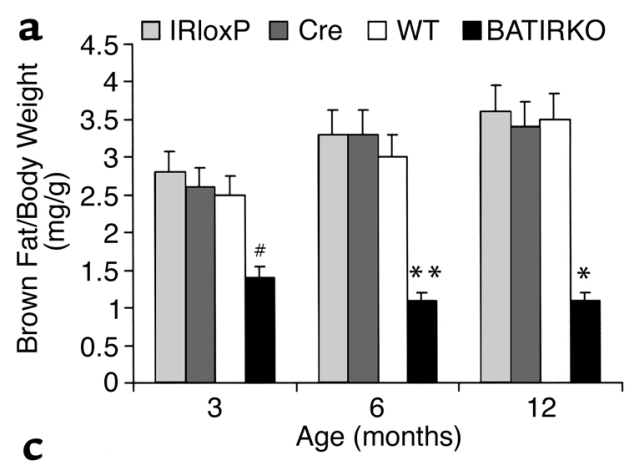

$\mathbf{C}$

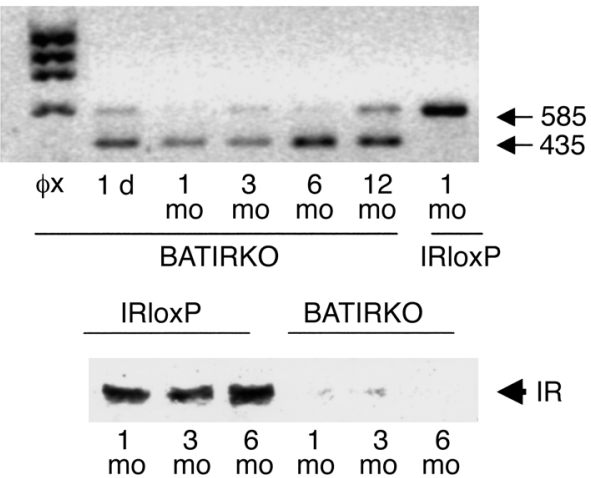

b

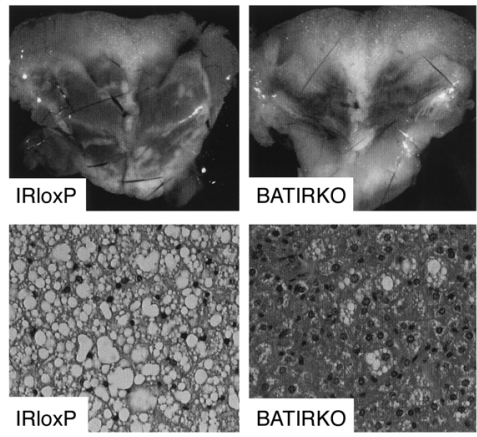

d

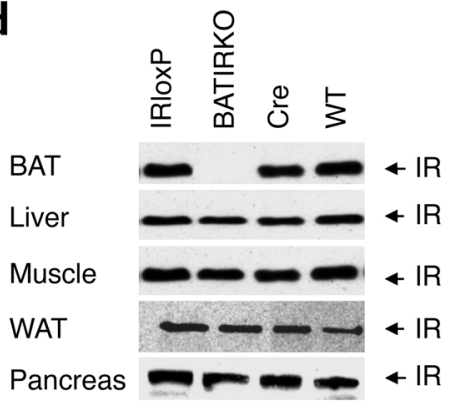

\section{Figure 2}

BAT content and IR expression. Interscapular brown fat was obtained from WT, Cre, IR/oxP, and BATIRKO mice at 3, 6, and 12 months of age. (a) BAT weight vs. body weight is represented. Results are expressed as a mean \pm SEM $(n=10-20) .{ }^{\#} P<0.00001$, BATIRKO vs. IR/oxP; ${ }^{*} P<0.0001$, BATIRKO vs. IR/oxP or Cre; ${ }^{*} P<0.01$, BATIRKO vs. IR/oxP or Cre or WT. Representative interscapular BAT from IR/oxP and BATIRKO mice at 6 months of age (b) (upper panel). Hematoxylin and eosin stain of BAT from IR/oxP and BATIRKO mice at 6 months of age (b) (lower panel). RT-PCR analysis of RNA prepared from BAT from BATIRKO mice at different ages ( 1 day and $1,3,6$, and 12 months) and IRloxP at 6 months of age, to study IR expression. The RT reaction was carried out as described in Methods. A larger band ( $585 \mathrm{bP}$ ) was observed in the IR/oxP lane, while a smaller band of 435 bp was observed in BATIRKO mice lanes, suggesting a recombination event (c) (upper panel). Protein extracts of BAT from IR/oxP and BATIRKO mice at different ages (1, 3, and 6 months) were subjected to immunoprecipitation with an $m A b$ against IR $\beta$-chain and analyzed by Western blot. This is representative of three experiments (c) (lower panel). Protein extracts from different tissues of 6-month-old WT, IR/oxP, Cre, and BATIRKO mice were immunoprecipitated with the same $A b$ and analyzed by Western blot. This is representative of three experiments (d). $\phi x$, DNA marker.

the expected ratio of $12.5 \%$ for a trait determined by two independent loci. This strategy of breeding $\left[\mathrm{IR}^{\mathrm{Lox} /+} ; \mathrm{UCP}-\right.$ 1 -Cre $\left.(\mathrm{T} /+) \times \mathrm{IR}^{\mathrm{Lox} /+}\right]$ was required to obtain all the control littermates necessary for the study [UCP-1-Cre $(\mathrm{T} /+)$, wild-type (WT), and IR ${ }^{\text {Lox/Lox }}$. To obtain enough numbers of mice with the same age and gender to carry out all these studies with BATIRKO and their three control mice, it was necessary to work with 12 individual crosses [IR ${ }^{\mathrm{Lox} / \mathrm{Lox}} ; \mathrm{UCP}-1-\mathrm{Cre}(\mathrm{T} /+) \times \mathrm{IR}^{\mathrm{Lox} /+}$ mice] during the 3 years of the study. All the mice used were in a mixture background of FVB/NJ, C57BL/6J, and $129 \mathrm{sv}$.

Although newborn BATIRKO mice were indistinguishable from the IR ${ }^{\text {Lox/Lox }}$ (IRloxP), UCP-1-Cre (Cre), and WT littermates, grew normally, and no differences were seen in young mice after weaning (data not shown), in adult BATIRKO mice the BAT appeared reduced in an age-dependent manner. Thus, the amount of interscapular BAT normalized by body weight (BAT/body weight mass ratio) was reduced by $50 \%$ in 3-month-old BATIRKO mice and by $75 \%$ in 6 - and 12 month-old BATIRKO mice as compared with controls (Figure 2a). This phenotype was shown by $100 \%$ of BATIRKO mice regardless of gender. By contrast, no significant differences were observed in the amount of white adipose tissue (WAT) as normalized by body weight (WAT/body weight-mass ratio) between BATIRKO mice and controls at any age studied (results not shown). Histological hematoxylin and eosin staining of interscapular BAT slides revealed a marked reduction of cell and fat droplet size and an increased eosinophilia in BATIRKO mice as compared with controls (Figure 2b, lower panel). These data are fully consistent with the marked interscapular BAT lipoatrophy induced in 6-month-old BATIRKO mice as compared with controls (Figure 2b, upper panel). However, no apoptosis was visualized by TUNEL assay in BAT sections as compared with controls (results not shown).

To assess the degree of recombination of the IR due to the excision by Cre recombinase of exon 4 (149 bp) floxed by loxP sequences, an age-dependent study by RT-PCR was performed. These data revealed that in control mice (IRloxP) only one band of larger size was observed. Conversely, in BATIRKO mice throughout development (from 1 day to 12 months) only one band of smaller size was visualized, a faint band of larger size due to the presence on non-brown adipocytes in the tissue also being present (Figure 2c, upper panel). Our data strongly suggest that the UCP1-Cre transgene is regulated in a very similar developmental fashion to the endogenous gene.

To validate the effect of the IR gene knockout on IR protein expression, protein extracts from BAT were subjected to immunoprecipitation with a monoclonal IRspecific antiserum, followed by Western blot analysis using a polyclonal IR-specific antiserum. IR expression was unaltered in BAT from the control IRloxP mice as compared with that from the WT animal, indicating that the introduction of the loxP sites in the IR allele or 
a

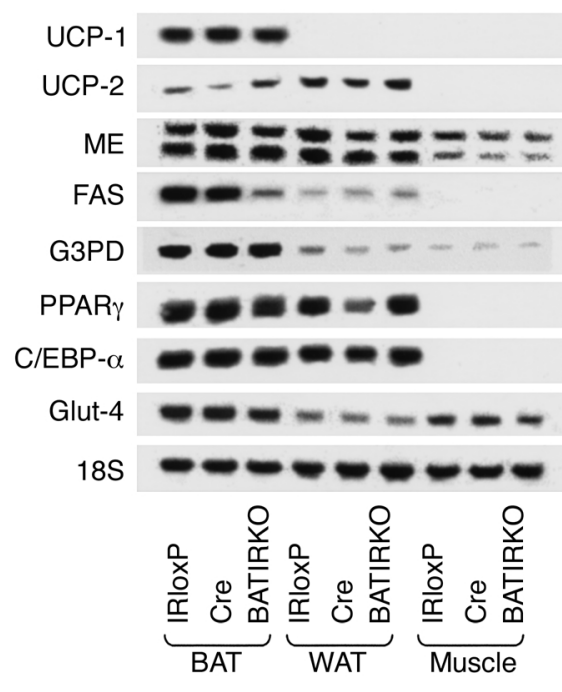

b

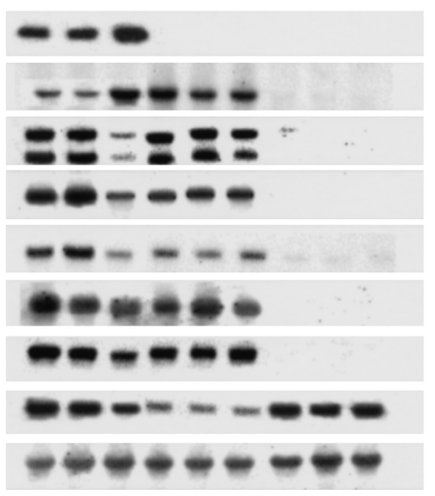

C

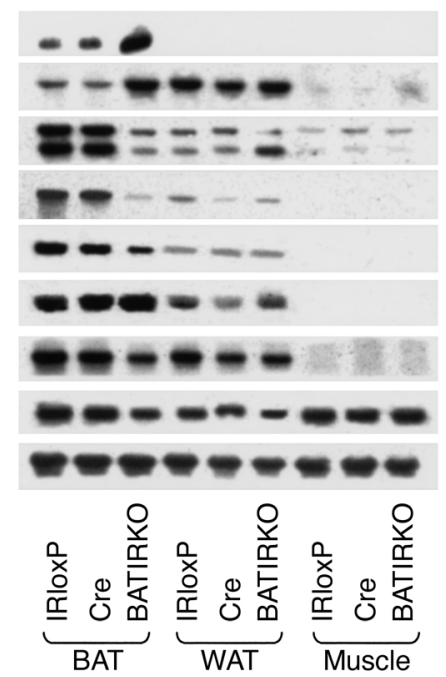

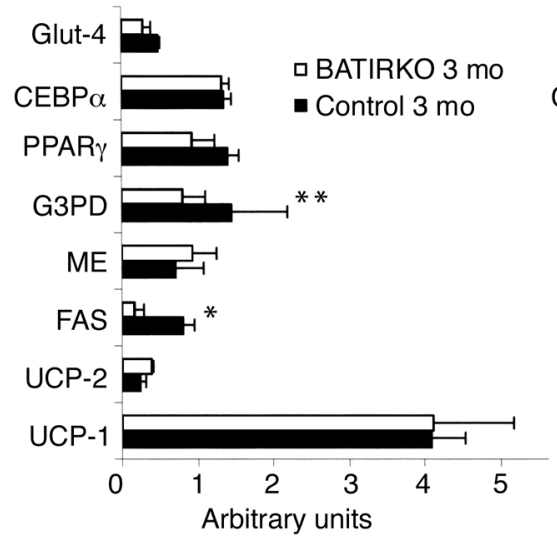
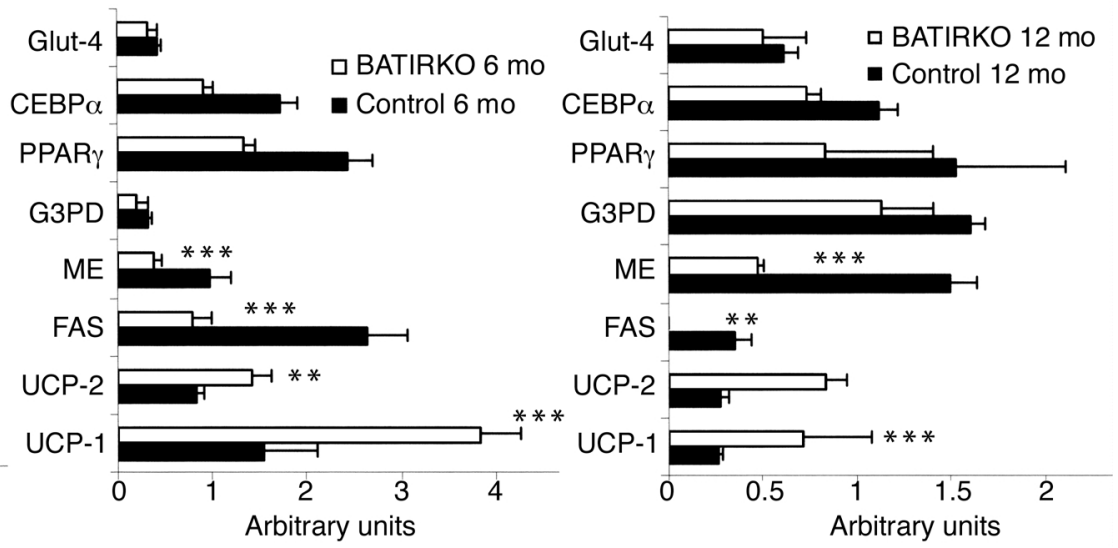

Figure 3

Gene expression throughout development. Skeletal muscle (Muscle), interscapular BAT, and WAT were obtained at 3 (a) (upper panel), 6 (b) (upper panel), and 12 months (c) (upper panel) of age from IRloxP, Cre, and BATIRKO mice. Total RNA was submitted to Northern blot analysis and hybridized with labeled UCP-1, UCP-2, ME, FAS, G3PD, PPAR $\gamma$, C/EBP- $\alpha$, or Glut-4. A final hybridization with 18S ribosomal RNA ( $r R N A)$ cDNA was performed for normalization. Densitometric analysis of the autoradiograms corresponding to BAT from controls (filled bars) and BATIRKO mice (open bars) after normalization of arbitrary units with the amount of 18S rRNA detected is shown in lower panels. Results are expressed as mean $\pm \operatorname{SEM}(n=5-6)$. ${ }^{*} P<0.05 ;{ }^{*} P<0.005 ;{ }^{*} P<0.001$ BATIRKO vs control. Autoradiograms are representative of five experiments.

Cre transgene under the UCP-1 promoter did not interfere with the endogenous IR expression (Figure 2d). By contrast, IR expression was reduced by $95 \%$ in BAT from BATIRKO mice (Figure 2d). The levels of IR protein in other tissues (liver, skeletal muscle, WAT, or pancreas) in 6-month-old BATIRKO mice as determined by Western blot analysis ranged from $83 \%$ to $120 \%$ of levels observed in WT, IRloxP, and Cre controls (Figure 2d). In addition, a time-course study showed the lack of expression of IR in BATIRKO mice at 1, 3, and 6 months, respectively, as compared with the corresponding control mice. (Figure 2c, lower panel). Thus, expression of the Cre transgene under control of the UCP-1 promoter was sufficient to direct recombination between the loxP sites flanking exon 4 (149 bp) of the IR with high efficiency in BAT and was highly effective in abolishing IR expression specifically in this tissue.
To assess the effect of IR on the expression of genes related to thermogenesis and lipogenesis, we performed Northern blot analysis on RNA from BAT, WAT, or skeletal muscle of BATIRKO mice and from IRloxP and Cre controls. A densitometric analysis was performed in mRNA expression of BAT from several genotypes throughout development (Figure 3, lower panels). Interestingly, at 3 months of age, expression of the major thermogenic protein, UCP-1, was normal in BAT of BATIRKO mice, despite the reduced BAT mass. Furthermore, as the BATIRKO mice aged, the expression of UCP-1 remained high as compared with IRloxP and Cre controls in which UCP-1 expression per microgram of RNA tended to decrease (Figure 3, a-c, lower panels). The expression of UCP-2 was also higher in brown fat of the BATIRKO mice as compared with controls throughout development and was even more evi- 


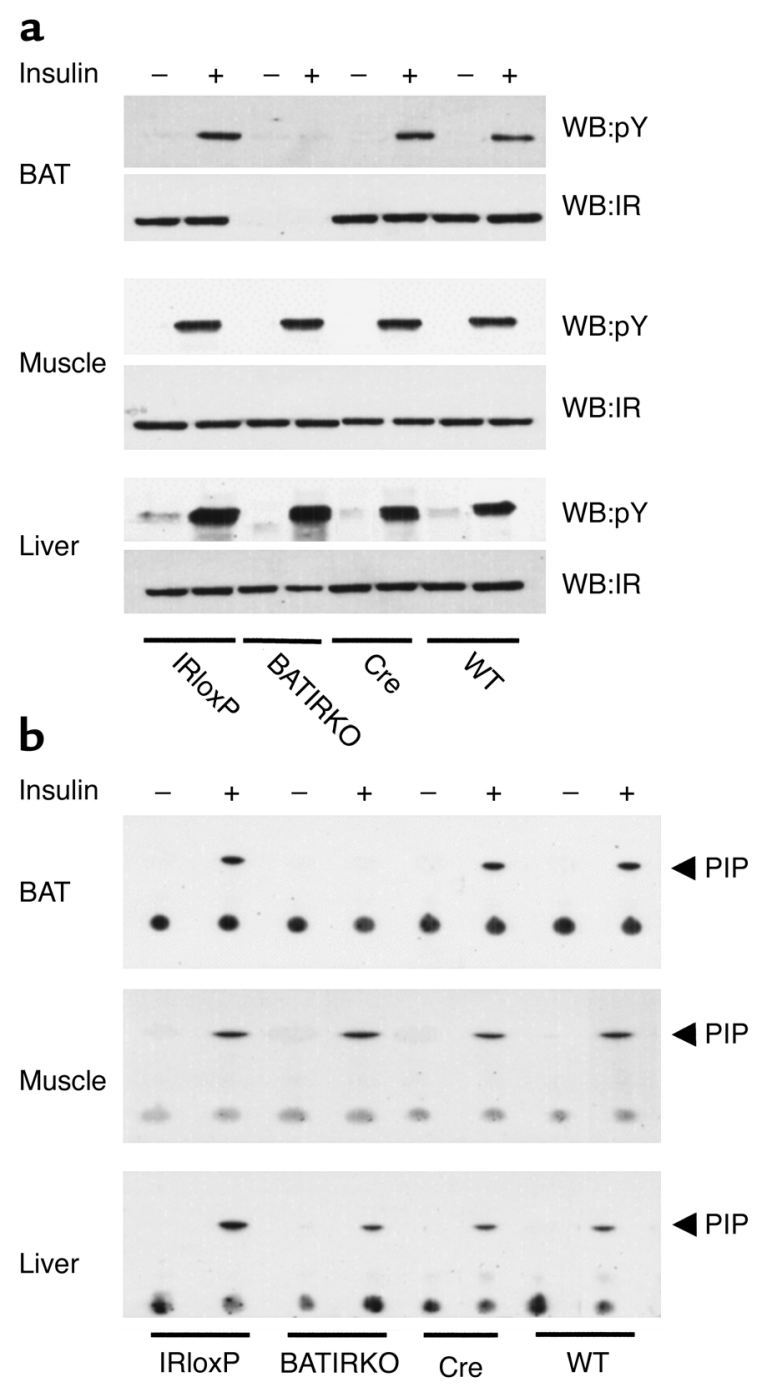

Figure 4

Insulin-stimulated tyrosine phosphorylation of the IR $\beta$-chain and PI 3-kinase activity. Six-month-old mice were anesthetized by intraperitoneal injection of pentobarbital and injected with either saline (-) or insulin $(+)$ via the inferior vena cava. Protein extracts from liver, muscle, and BAT were subjected to immunoprecipitation with the monoclonal anti-IR Ab (a) or with a monoclonal anti-Tyr (P) Ab (b). The resulting immune complexes were separated by SDS-PAGE and analyzed by Western blot with the anti-Tyr $(P)$ Ab or with the polyclonal anti-IR Ab (a), or washed and immediately used for an in vitro PI 3kinase assay (b). This is representative of three experiments. WB, Western blot; PY, phospho-tyrosine; PIP, phosphatidylinositol phosphate.

dent in 12-month-old than in 3-to 6-month-old mice (Figure 3c, lower panel). We have shown previously that BAT undergoes a complete adipogenic program of gene expression in which insulin is the main signal involved in induction of lipogenic genes, such as FAS, glycerol3-phosphate dehydrogenase (G3PD), ME, and the Glut-4 (21). Densitometric analysis also revealed a marked decrease in FAS expression in remnant BAT from 3- to 12-month old BATIRKO mice as compared to controls. A decrease was also observed in ME and to a lesser extent in G3PD and Glut-4 expression in BATIRKO mice as compared with controls (Figure 3, lower panels). These changes in gene expression were age dependent, with the largest effect being observed in 6- to 12-month-old mice (Figure 3, b and c, lower panels). There was also a slight decrease in the expression of PPAR $\gamma$ and a marked decrease in the CCAAT/enhancer-binding protein- $\alpha(\mathrm{C} / \mathrm{EBP}-\alpha)$ in 6and 12-month old BATIRKO mice as compared with controls (Figure 3, b and c, lower panels).

Insulin-stimulated signaling events in liver, muscle, and BAT from WT, IRloxP, Cre, and BATIRKO mice. To assess the molecular consequences of BAT-specific knockout of IR on insulin signaling, we compared the early steps of insulin signaling in liver, skeletal muscle, and BAT of WT, IRloxP, Cre, and BATIRKO mice. To investigate IR phosphorylation, mice were starved overnight and injected with either saline or insulin (5 U) via the inferior vena cava. Protein extracts were prepared from liver, muscle, and BAT and subjected to immunoprecipitation with a monoclonal anti-IR Ab followed by Western blot analysis with an anti-Tyr (P) Ab. Insulin rapidly stimulated tyrosine phosphorylation of the IR in liver and muscle from the WT, IRloxP, Cre, and BATIRKO mice (Figure 4a). In contrast, no insulininduced tyrosine phosphorylation of the IR was observed in the BAT of BATIRKO mice. Reblotting of these membranes with an anti-IR Ab revealed that, as expected, IR expression was absent in the BAT of BATIRKO mice, but remained normally expressed in liver and muscle of these animals (Figure 4a).

To determine the effect of IR deletion on the activation of phosphatidylinositol (PI) 3-kinase, WT, IRloxP, $\mathrm{Cre}$, and BATIRKO mice were injected with insulin. Protein extracts from various tissues were immunoprecipitated with an anti-Tyr $(\mathrm{P}) \mathrm{Ab}$ and immediately used for an in vitro PI 3-kinase assay as described in Methods. Insulin-stimulated PI 3-kinase activation was normal in liver, muscle, and BAT from WT, IRloxP, and Cre mice (Figure 4b). Again, as expected, BAT from BATIRKO mice did not show PI 3-kinase activity in response to insulin, while insulin-induced PI 3-kinase activity in liver and muscle of these animals remained normal as compared with WT, IRloxP, and Cre controls (Figure $4 \mathrm{~b})$. These data reinforce the fact that the knockout of the IR was both tissue specific and quite complete.

BATIRKO mice exhibited an age-dependent impaired glucose tolerance, without insulin resistance. To determine the physiological consequence of tissue-specific inactivation of the IR in brown fat, glucose disposal was assessed by intraperitoneal injection of glucose $(2 \mathrm{~g} / \mathrm{kg}$ body weight) following an overnight fast. Upon glucose challenge, at 3 months of age both male and female BATIRKO mice showed a normal fasting blood glucose concentration and normal glucose tolerance as compared with their controls (Figure 5, a and b). However, beyond 6 months of age BATIRKO males showed fasting hyperglycemia. In addition, glucose tolerance was severely impaired in BATIRKO males at 6 months of age as compared with their controls, and by 12 months of age glucose levels following intraperitoneal glucose 

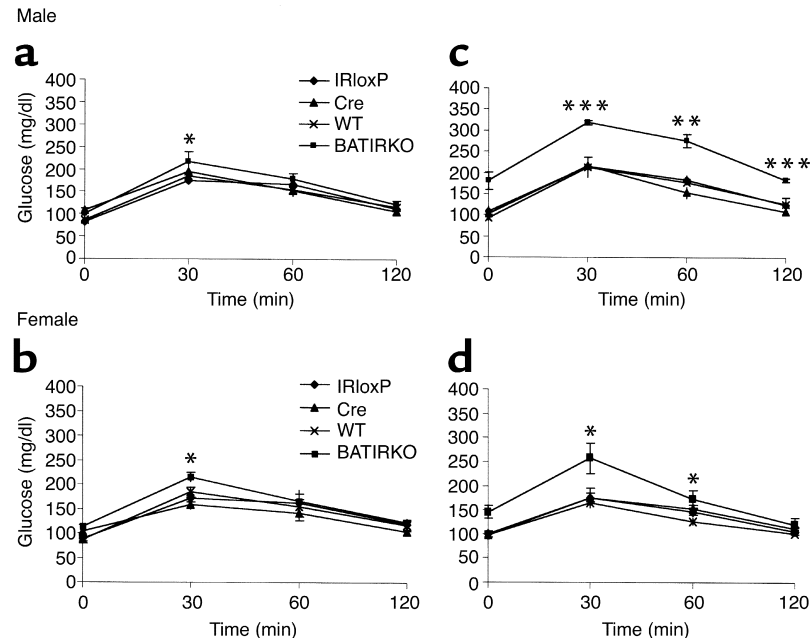

Figure 5

BATIRKO mice demonstrate a progressive glucose intolerance. The ability to handle a glucose load was assessed by carrying out a glucose tolerance test at 3, 6, and 12 months of age in WT $(\times)$, Cre (triangles), IR/oxP (diamonds), and BATIRKO (squares) male (a, c, and e) and female (b, $\mathbf{d}$, and $\mathbf{f}$ ) mice. An age-dependent glucose intolerance was observed in both male and female mice. Results are expressed as mean $\pm \operatorname{SEM}(n=10-20) .{ }^{*}{ }^{*} P<0.0005 ;{ }^{*} P<0.001 ;{ }^{*} P<0.005 ;{ }^{*} P<0.05$; BATIRKO vs. WT or IR/oxP or Cre.

challenge were from to 1.5 -fold to twofold higher in BATIRKO mice than in controls (Figure 5, $\mathrm{c}$ and e). BATIRKO females beyond 6 months of age also showed mild hyperglycemia and moderately impaired glucose tolerance as compared with controls. By 12 months of age, glucose levels 30 minutes after intraperitoneal glucose were 1.5 -fold higher in BATIRKO mice than in their controls (Figure 5, d and f). Overall, this diabetic phenotype was shown by $60 \%$ of BATIRKO mice. Intravenous insulin tolerance (ITT) testing $(1 \mathrm{U} / \mathrm{kg}$ body weight), on the other hand, indicated that both 6month-old male and female BATIRKO mice showed the same glucose disposal rate in response to insulin than controls (results not shown).

BATIRKO mice exbibited an insulin-secretion defect. The normal ITT, but abnormal glucose tolerance test, suggested that the defect in glucose homeostasis might be secondary to altered insulin secretion, rather than altered insulin action. Indeed, acute glucose-stimulated insulin secretion ( $3 \mathrm{~g}$ of glucose $/ \mathrm{kg}$ body weight) was dramatically impaired in fasted 6-month-old male BATIRKO mice as compared with controls (Figure 6a). At this stage, fasted male BATIRKO mice were hyperglycemic (Figure 6b, upper panel) and showed a marked hypoinsulinemia (Figure 6b, lower panel), without significantly altered levels of free fatty acids $(0.52 \pm 0.07$ $\mathrm{mM}$ in BATIRKO vs. $0.43 \pm 0.07 \mathrm{mM}$ in controls) and triglycerides $(87.8 \pm 4.4 \mathrm{mg} / \mathrm{dl}$ in BATIRKO vs. $96.5 \pm 5.8$ $\mathrm{mg} / \mathrm{dl}$ in controls). However, fed male BATIRKO mice showed similar blood glucose concentration to controls, plasma insulinemia being slightly lower (Figure 6b).

Histologic examination of islet morphology revealed that BATIRKO diabetic mice showed a decrease in the islet size. However, islet size was quite similar in
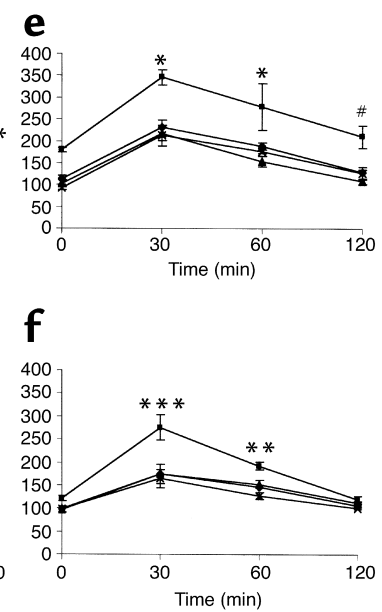

BATIRKO nondiabetic mice as compared with controls (Figure 6c, upper panels). Overall, quantitative analysis of pancreatic sections revealed that male BATIRKO mice showed around $35 \%$ decrease in beta islet mass (area) as compared with controls (Figure $6 c$, lower panel). In addition, TUNEL assay revealed the absence of apoptotic $\beta$ cells in BATIRKO pancreatic sections as compared with controls (results not shown).

A 1-hour time course of insulin secretion in isolated islets upon $16.7 \mathrm{mM}$ glucose stimulation was also studied (Figure 6d). Thus, insulin secretion was blunted in isolated islets from BATIRKO male mice as compared with controls. This effect is entirely consistent with data from the acute insulin-secretion test seen above. However, the Lox-P-IR recombination assay performed by RT-PCR showed no recombination in islet RNA from BATIRKO mice as compared with controls (Figure 6e, left panel), suggesting that the defect in the insulin secretion in BATIRKO mice seems to be an indirect effect. Northern blot analysis of islet RNA, normalized by $18 \mathrm{~S}$ rRNA expression, pointed out that the insulin mRNA expression was quite similar in BATIRKO mice as compared with control. More importantly, the UCP-2 mRNA expression did not show significant differences between BATIRKO and control mice (Figure 6e, right panels).

\section{Discussion}

Type 2 diabetes is characterized by defects in both insulin action and in insulin secretion $(1,2)$. The peripheral insulin resistance has been demonstrated in muscle, WAT, and liver; however, little is known about the potential role of insulin action or insulin resistance in BAT in type 2 diabetes. Development of BATIRKO mice has revealed two potentially important aspects of insulin resistance in this tissue with respect to whole body glucose homeostasis. First, insulin plays an important role in development or maintenance of BAT. Thus, mice lacking IR in BAT exhibit profound brown fat atrophy in an age-dependent manner. This phenotype is further reflected by a profound reduction in the expression of the lipogenic genes seen in the remnant interscapular BAT. Although the exact signaling pathway by which IR signaling plays a role in adipogenesis in brown fat is probably multifold, our data indicate that the expression of C/EBP- $\alpha$ in BAT is strongly dependent on the IR signaling throughout development. By contrast, expression of PPAR $\gamma$, the other tran- 
a

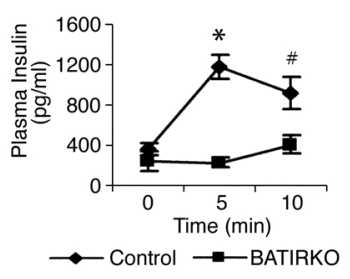

b
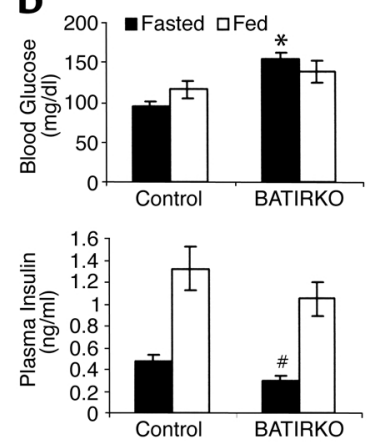

$\mathbf{C}$

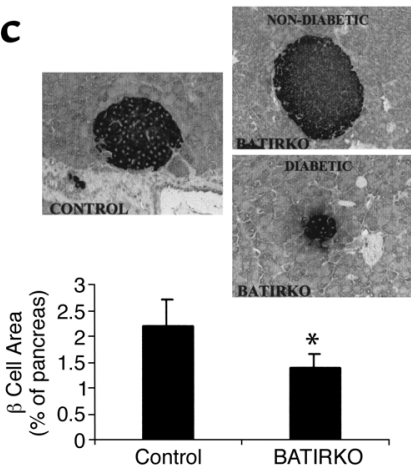

Control BATIRKO d

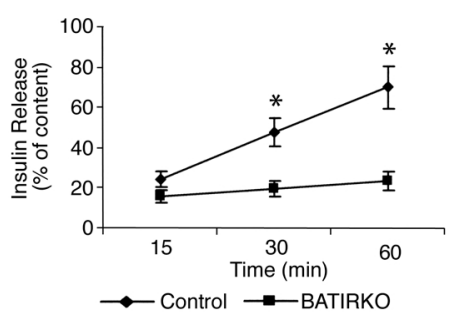

e

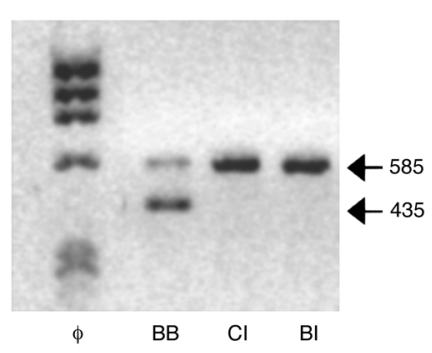

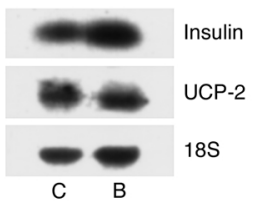

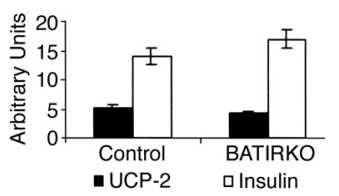

Figure 6

BATIRKO male mice show an insulin-secretion defect. A representative insulin-secretion test is shown. Results are expressed as mean \pm SEM $(n=7-12)$. ${ }^{*} P<0.001 ;{ }^{\#} P<0.005$ (a). Blood glucose level (b) (upper panel) and blood insulin level (b) (lower panel) were determined in fasted and fed control and BATIRKO mice. Results are expressed as mean \pm SEM $(n=25-30),{ }^{*} P<0.000001,{ }^{*} P<0.01$ BATIRKO vs. control. Islet morphology was studied by immunohistochemical analysis in BATIRKO and control mice, as shown in Methods. Representative pancreatic sections from control and diabetic and nondiabetic 6-month-old BATIRKO mice stained for insulin are shown (c) (upper panels). $\beta$-cell area was measured by immunohistochemical analysis. Results are expressed as the percentage of the total surveyed area containing cells positive for insulin and are mean $\pm \operatorname{SEM}(n=10) .{ }^{*} P<0.01$. Both diabetic and nondiabetic animals were studied (c) (lower panel). Insulin secretion in control and BATIRKO isolated islets is shown. Results are expressed as mean $\pm \operatorname{SEM}(n=4)$. ${ }^{*} P<0.05(\mathbf{d})$. RT-PCR analysis of total RNA from BAT of BATIRKO mice (BB) and islets from control (CI) and BATIRKO mice (BI) was performed, and IR expression was studied (e) (left panel). Total islet RNA from control (C) and BATIRKO (B) mice was submitted to Northern blot analysis and hybridized with labeled insulin and UCP-2 cDNAs. A representative experiment out of three is shown. Densitometric analysis was performed using $18 \mathrm{~S}$ ribosomal rRNA for normalization (e) (right panels).

scriptional factor essential for induction of the adipocyte phenotype (24-27), is only minimally reduced in BATIRKO adipose tissue. The loss of brown fat mass might also be due to an increase in the rate of apoptosis related to the lack of IR and its PI 3-kinase/Akt downstream survival signaling. However, our data rule out this possibility. Interestingly, the lack of IR leads to the overexpression of the UCP-1 and also UCP-2 in the remnant BAT from BATIRKO as compared with controls. These data could be interpreted as a form of compensatory mechanism for the brown fat lipid content and mass loss observed in BATIRKO and may result in a potential increase in the thermogenic capacity of the remnant BAT that may account for the lean phenotype of BATIRKO mice compared with controls.

The second phenotype observed in the BATIRKO mice is an altered regulation of glucose homeostasis. Thus, the lack of IR in the BAT leads to an overall moderate reduced percentage of beta cell mass, a significant decrease in basal insulin, and a marked insulin-secretion defect in response to glucose in vivo and in isolated islets. This leads to a diabetic phenotype with fast- ing hyperglycemia and impaired glucose tolerance. This phenotype becomes apparent in an age-dependent manner, suggesting that the diabetic phenotype be related to brown fat atrophy. Exactly what accounts for the moderate decrease in beta cell mass and insulin levels is unclear. A defect in insulin secretion and progressive glucose intolerance is observed in mice with a $\beta$ cell-specific knockout of the IR (19). However, UCP-1 is not expressed in islets, so this is unlikely to represent some nonspecificity of the knockout itself. In fact, the recombination assay proved that the expression of IR in $\beta$ islets from BATIRKO mice was unaltered, strongly suggesting an indirect mechanism. This also occurs with no change in the levels of triglycerides and free fatty acids as compared with controls, suggesting this is not some form of lipotoxicity. Even further, the expression of UCP-2, a mitochondrial protein that regulates the insulin secretion through ATP/ADP cellular content (28), does not change in $\beta$ islets from BATIRKO mice. However, an alteration in the biochemical machinery involved in the insulin secretion response to glucose cannot be totally ruled out. In addi- 
tion, high UCP-2 content in BATIRKO mice islets might protect these cells against oxidants and apoptosis (29). Rather, our data suggest a direct or indirect functional link between brown fat and beta cell development. Thus, the reduction in the beta cell mass and the defect in the insulin secretion in response to glucose, might be related to the dysregulation of an adipoinsular axis due to the lack of IR in BAT and its atrophy. In fact, mice lacking $\beta_{3}$ adrenoceptors in white and brown adipose tissues failed to respond to $\beta_{3}$-agonist treatment in increasing insulin secretion. However, the transgenic re-expression of $\beta_{3}$ adrenoceptors in BAT only failed to restore the $\beta_{3}$-agonist-mediated insulin secretion, indicating that the role of WAT is critical (30). This situation is somewhat different from that which occurs in A-ZIP-F-1 transgenic mice, where the lack of white fat can cause diabetes (31). Thus, BATIRKO mice show brown fat atrophy and also a diabetic phenotype without implying insulin resistance, whereas in A-ZIP-F-1 mice the lack of white fat leads to an accumulation of triglyceride in muscle and liver, resulting in a severe insulin resistance in those tissues and hyperinsulinemia (32). More importantly, A-ZIP/F-1 lipoatrophic mice lack the $\beta_{3}$-agonist-mediated insulin secretion. These data also suggest a link between adipocytes and $\beta$-cell function (33).

Whatever the mechanism, BATIRKO mice reveal two previously unrecognized aspects of the physiological brown fat homeostasis: first, the leading role played by IR in the adipogenesis of brown fat throughout development and, second, the role of BAT in the regulation of insulin secretion and glucose homeostasis.

\section{Acknowledgments}

We wish to give special thanks to J.A. López for his technical skill with brown fat histology and the immunohistochemistry of $\beta$ islets; to the personnel of the Animal House of The Jackson Laboratory and Universidad Complutense, Madrid, Spain; and to Mercedes López for her assistance with mice genotyping. This work was supported by grant DK-3320 from the NIH and grant PM 98/0087 from the Ministerio de Ciencia y Tecnologia, Spain.

1. Warram, J.H., Martin, B.C., Krolewski, A.S., Soeldner, J.S., and Kahn, C.R. 1990. Slow glucose removal rate and hyperinsulinemia precede the development of type II diabetes in the offspring of diabetic parents. Ann. Intern. Med. 113:909-915.

2. Virkamaki, A., Ueki, K., and Kahn, C.R. 1999. Protein-protein interaction in the insulin signaling and the molecular mechanism of insulin resistance. J. Clin. Invest. 103:931-943.

3. Martin, B.C., Warram, J.H, Krolewski, A.S., Soeldner, J.S., and Kahn, C.R. 1992. Role of glucose and insulin resistance in development of type II diabetes mellitus: results of 25 year follow-up study. Lancet. 340:925-929.

4. Kahn, C.R. 1994. Insulin action, diabetogenes, and the cause of type II diabetes. Diabetes. 43:1066-1084.

5. Caro, J.F., et al. 1987. Insulin receptor kinase in human skeletal muscle from obese subjects with and without non-insulin dependent diabetes. J. Clin. Invest. 79:1330-1337.
6. Olefsky, J.M., and Nolan, J.J. 1995. Insulin resistance and non-insulindependent diabetes mellitus: cellular and molecular mechanisms. Am.J. Clin. Nutr. 61:980s-986s.

7. Kerouz, N.J., Horsch, S., Pons, S., and Kahn, C.R. 1997. Differential regulation of insulin on insulin receptor substrates-1 and -2 (IRS-1 and IRS2) and phophatidylinositol 3-kinase isoforms in liver and muscle of obese diabetic (ob/ob) mouse. J. Clin. Invest. 100:3164-3172.

8. Rondinone, C.M., et al. 1997. Insulin receptor substrate (IRS) 1 is reduced and IRS-2 is the main docking protein for phosphatidyl inositol 3-kinase in adipocytes from subjects with non-insulin-dependent diabetes mellitus. Proc. Natl. Acad. Sci. USA. 94:4171-4175.

9. Accili, D., et al. 1996. Early neonatal death in mice homozygous for a null allele of the insulin receptor gene. Nat. Genet. 12:106-109.

10. Joshi, R.L., et al. 1996. Targeted disruption of the insulin receptor gene in the mouse results in neonatal lethality. EMBO J. 15:1542-1547.

11. Araki, E., et al. 1994. Alternative pathways of insulin signaling in mice with targeted disruption of the IRS-1 gene. Nature. 372:186-190.

12. Tamemoto, H., et al. 1994. Insulin resistance and growth retardation in mice lacking insulin receptor substrate-1. Nature. 372:182-186.

13. Withers, D.J., et al. 1998. Disruption of IRS-2 causes type- 2 diabetes in mice. Nature. 391:900-903.

14. Withers, D.J., et al. 1999. Irs-2 coordinates Igf- 1 receptor-mediated betacell development and peripheral insulin signaling. Nat. Genet. 23:32-40.

15. Bruning, J.C., et al. 1997. Development of a novel polygenic model of NIDDM in mice heterozygous for IR and IRS-1 null alleles. Cell. 88:561-572.

16. Kido, Y., et al. 2000. Tissue-specific insulin resistance in mice with mutations in the insulin receptor, IRS-1, and IRS-2. J. Clin. Invest. 105:199-205.

17. Bruning, J.C., et al. 1998. A muscle-specific insulin receptor knockout exhibits features of the metabolic syndrome of NIDDM without altering glucose tolerance. Mol. Cell. 2:559-569.

18. Michael, M.D., et al. 2000. Loss of insulin signaling in hepatocytes leads to severe insulin resistance and progressive hepatic dysfunction. Mol. Cell. 6:87-97.

19. Kulkarni, R.N., et al. 1999. Tissue-specific knockout of the insulin receptor in pancreatic $\beta$ cells creates an insulin secretory defect similar to that in type II diabetes. Cell. 96:329-339.

20. Bruning, J.C., et al. 2000. Role of brain insulin receptor in control of body weight and reproduction. Science. 289:2122-2125.

21. Chomczynski, P., and Sacchi, N. 1987. Single-step method of RNA isolation by acid guanidinium thiocyanate-phenol-chloroform extraction. Anal. Biochem. 162:156-159.

22. Teruel, T., Valverde, A.M., Benito, M., and Lorenzo, M. 1996. Insulinlike growth factor-I and insulin induce adipogenic-related gene expression in foetal brown adipocyte primary cultures. Biochem. J. 319:627-632.

23. Valverde, A.M., Lorenzo, M., Pons, S., White, M.F., and Benito, M. 1998. IRS-1 and IRS-2 differential signaling in the insulin/IGF-I pathways in fetal brown adipocytes. Mol. Endocrinol. 12:688-697.

24. Brun, R.P., Kim, J.B., Hu, E., Altiok, S., and Spiegelman, B.M. 1996. Adipocyte differentiation: a transcriptional regulatory cascade. Curr. Opin. Cell Biol. 8:826-883.

25. Rosen, E.D., et al. 1999. PPAR gamma is required for the differentiation of adipose tissue in vivo and in vitro. Mol. Cell. 4:611-617.

26. Wu, Z., Puigserver, P., and Spiegelman, B.M. 1999. Transcriptional activation of adipogenesis. Curr. Opin. Cell Biol. 11:689-694.

27. Wu, Z., et al. 1999. Cross-regulation of C/EBP alpha and PPAR gamma controls the transcriptional pathway of adipogenesis and insulin sensitivity. Mol. Cell. 3:151-158.

28. Zhang, C.Y., et al. 2001. Uncoupling protein-2 negatively regulates insulin secretion and is the major link between obesity, $\beta$ cell dysfunction, and type-2 diabetes. Cell. 105:745-755.

29. Li, L.X., Skorpen, F., Egeberg, K., Jorgensen, I.H., and Grill, V. 2001. Uncoupling protein-2 participates in cellular defense against oxidative stress in clonal beta-cells. Biochem. Biophys. Res. Commun. 282:273-277.

30. Grujic, D., et al. 1997. $\beta_{3}$-adrenergic receptors on white and brown adipocytes mediate $\beta_{3}$-selective agonist-induced effects on energy expenditure, insulin secretion and food intake. J. Biol. Chem. 28:17686-17693.

31. Moitra, J., et al. 1998. Life without white fat: a transgenic mouse. Genes Dev. 12:3168-3181.

32. Kim, J.K., Gavrilova, O., Chen, Y., Reitman, M.L., and Shulman, G.I. 2000. Mechanism of insulin resistance in A-ZIP/F-1 fatless mice. J. Biol. Chem. 275:8456-8460.

33. Gavrilova, O., Marcus-Samuels, B., and Reitman, M.L. 2000. Lack of responses to a beta3-adrenergic agonist in lipoatrophic A-ZIP/F-1 mice. Diabetes. 49:1910-1916. 